\title{
Spektroskopischer Nachweis eines Bis(amino)silylens
}

\author{
M. Veith*a , E. Werle ${ }^{a}$, R. Lisowsky ${ }^{a}$, R. Köppe ${ }^{\text {b }}$ und H. Schnöckel * b \\ Institut für Anorganische Chemie der Universität des Saarlandes ${ }^{\text {a }}$, \\ W-6600 Saarbrücken
}

Institut für Anorganische Chemic der Universität München ${ }^{b}$, W-8000 München 2

Eingegangen am 17. Januar 1992

Key Words: Silicon(IV) diazide / Photolysis / Matrix isolation / Silylene, bis(amino)-

\section{Spectroscopic Identification of a Bis(amino)silylene}

The photolysis of the silicon diazide $\mathbf{3 a}$ in benzene solution and in an Ar matrix is described. Both irradiations cause the elimination of 3 equivalents of $\mathrm{N}_{2}$. Loss of $\mathrm{N}_{2}$ from $3 \mathrm{a}$ in benzene leads to the formation of the analytically investigated product or products 4 of uncertain structure. However, the matrix photolysis of $\mathbf{3 a}$ results in a compound which is stable up to $77 \mathrm{~K}$ and has been identified as the bis(amino)silylene $2 \mathrm{~d}$ by comparison of its IR spectra with those of the homologous Sn and Ge compond.

Substitution der Wasserstoff-Atome im Bis(amino)silan $1^{[1]}$ durch zweiwertige Elemente der 4. Hauptgruppe führt zu cyclischen Carben-analogen Amiden 2a-2c. Diese Verbindungen sind bei Raumtemperatur stabil und liegen in nicht koordinierenden Lösemitteln monomer vor ${ }^{[2 \mathrm{a}, \mathrm{b}]}[\mathrm{Gl}$. (1)].
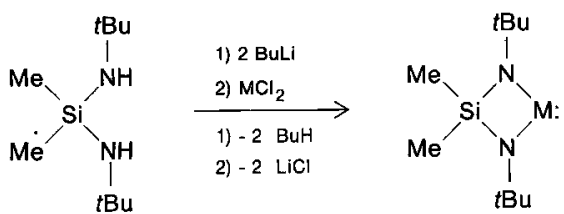

1

\begin{tabular}{l|lll}
$\mathbf{2}$ & $\mathbf{a}$ & $\mathbf{b}$ & $\mathbf{c}$ \\
\hline $\mathrm{M}$ & $\mathrm{Ge}$ & $\mathrm{Sn}$ & $\mathrm{Pb}$
\end{tabular}

In der vorliegenden Arbeit wird der Frage nachgegangen, ob auch zweiwertiges Silicium auf diese Weise stabilisiert werden kann. Bisher ist ein solches Bis(amino) silylen unbekannt; in jüngster Zeit sind die Synthesen von zwei bei Normalbedingungen stabilen Verbindungen beschrieben worden, in denen Silicium formal zweiwertig vorliegt, $\mathrm{Si}\left(\mathrm{Me}_{5} \mathrm{C}_{5}\right)_{2}$ und $\mathrm{Si}\left[\mathrm{C}\left(\mathrm{PMe}_{2}\right)_{3}\right]_{2}{ }^{[3-5]}$.

Als Edukt zur Darstellung eines Bis(amino)silylens 2d $(\mathrm{M}=\mathrm{Si})$ schien das vor kurzem von uns synthetisierte Si-<smiles></smiles>
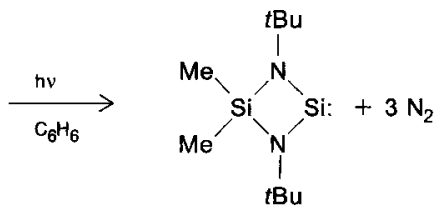

2d lylazid $3 \mathbf{a}^{[6]}$ besonders geeignet. Die thermolytische oder photochemische Abspaltung von Distickstoff sollte zumindest intermediär zur Zielverbindung 2d führen [Gl. (2)].

Führt man eine Photolyse an der Verbindung 3a in Benzol durch, so erhält man neben der erwarteten Menge an Distickstoff als isolierbares Produkt nicht 2d, sondern eine Verbindung bzw. ein Verbindungsgemisch der analytischen Zusammensetzung $\mathrm{Me}_{2} \mathrm{Si}(\mathrm{N} t \mathrm{Bu})_{2} \mathrm{Si} \cdot 0.6 \mathrm{C}_{6} \mathrm{H}_{6}$ (4) [Gl. (3)].

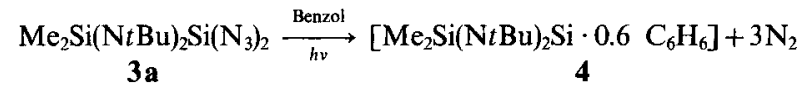

4 ist röntgenamorph, und das ${ }^{1} \mathrm{H}-\mathrm{NMR}-\mathrm{Spektrum}$ in Benzol enthält eine Vielzahl von Resonanz-Signalen, was auf ein Gemisch bzw. ein Oligomer oder ein Polymer hinweist. Weiterhin ist $4 \mathrm{im}$ Vakuum $\left(10^{-3}\right.$ Torr $)$ bis $70^{\circ} \mathrm{C}$ nicht flüchtig; danach zersetzt sich die Substanz, was auch im Massenspektrum zu erkennen ist. Bei $55^{\circ} \mathrm{C}(40 \mathrm{eV})$ läßt sich bereits das Cyclodisilazan $\mathrm{Me}_{2} \mathrm{Si}(\mathrm{NtBu})_{2} \mathrm{SiMe}_{2}$ neben anderen Silazanen nachweisen. Im IR-Spektrum von 4 sind keine AzidBanden mehr vorhanden; im Vergleich mit den Spektren von $2 \mathrm{~d}$ und $3 \mathbf{a}^{[6]}$ sind die Absorptions-Banden verschoben, ohne daß neue auftreten. Es kann mit Sicherheit nicht entschieden werden, ob das in der Elementarenalyse gefundene Benzol bzw. der Kohlenwasserstoff chemisch gebunden oder nur als eingeschlossenes Lösungsmittel vorliegt.

Auch die analoge Photolyse des von uns dargestellten Germaniumdiazids $\mathrm{Me}_{2} \mathrm{Si}(\mathrm{N} t \mathrm{Bu})_{2} \mathrm{Ge}\left(\mathrm{N}_{3}\right)_{2}(3 \mathrm{~b})^{\left[{ }^{[]}\right]}$liefert trotz nachgewiesener Abspaltung von $\mathrm{N}_{2}$ nicht das bekannte und stabile Bis(amino)germylen $\mathbf{2 a}$, sondern nicht auftrennbare Gemische von bisher unbekannten Substanzen. Die Photolyse der Diazide 3a bzw. 3b in Benzol ist offensichtlich zur Darstellung der niedervalenten Verbindungen 2a bzw. 2d ungeeignet. Möglicherweise treten bei Belichtung mit energiereicher Strahlung reaktive Intermediate auf, die mit 
sich selbst oder mit dem Lösemittel reagieren, bevor sie ein Bis(amino)carben-analoges Molekül bilden können. Vergleicht man diese Befunde mit der analogen Photolyse von Bis(tert-butyl)silyldiazid in Gegenwart von unterschiedlichen kleinen Molekülen, so werden die hier gefundenen Ergebnisse verständlich ${ }^{[8]}$. Um solche und ähnliche Sekundärreaktionen zu unterdrücken, haben wir die Bestrahlung des Silylazids 3a in einer Argon-Matrix durchgeführt (siehe auch Darstellung von Silanitrenen ${ }^{[9]}$ ).

Hierzu wurde das Silylazid gemeinsam mit einem Überschuß an Argon auf einer Kältefläche kondensiert. AnschlieBend wurde die Matrix mit dem Gesamt-Spektrum einer Hg-Lampe bestrahlt, wobei sich mit Hilfe eines FT-IR-Gerätes der Reaktionsablauf verfolgen ließ. Bereits nach einer Belichtungsdauer von 10 Minuten ist kein Edukt mehr nachzuweisen; das Silylazid 3a hat sich vollständig zersetzt. Abb. 1 gibt eine Zusammenstellung der IR-Spektren der Verbindungen 2a, $\mathbf{2 b}$ und des Photolyseproduktes von 3a wieder. Ein Vergleich der Spektren zeigt deutlich, daß Lagen und Intensitäten der stärksten Absorptionsbanden übereinstimmen. Ferner weisen einige Absorptionsbanden mit steigender Masse des Elements $\mathbf{M}$ in $\mathrm{Me}_{2} \mathrm{Si}(\mathrm{N} t \mathrm{Bu})_{2} \mathrm{M}$ : die zu erwartende geringfügige Verschiebung zu kleineren Wellen-

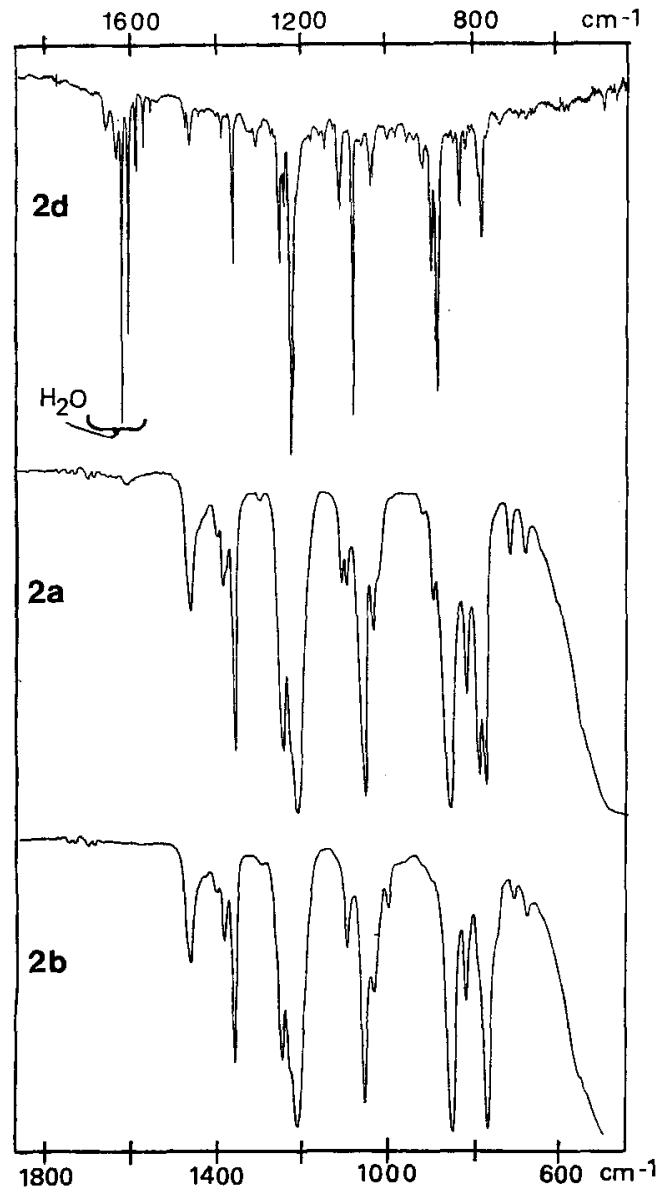

Abb. 1. Die IR-Spektren von $\mathrm{Me}_{2} \mathrm{Si}(\mathrm{N} t \mathrm{Bu})_{2} \mathrm{M}[\mathrm{M}=\mathrm{Si}(2 \mathrm{~d}$; Matrix), Ge $(2 \mathbf{a} ;$ Film), Sn (2b; Film)] im Bereich zwischen $\tilde{v}=1800$ und $400 \mathrm{~cm}^{-1}$; die zusätzlichen Schwingungsbanden zwischen $\tilde{v}=1700$ und $1500 \mathrm{~cm}^{-1} \mathrm{im}$ Spektrum von $2 \mathrm{~d}$ rühren von aufkondensiertem Wasser und dienen gleichzeitig zur Eichung zahlen auf (vgl. Exp. Teil). Auch die schwächeren Absorptionsbanden im Spektrum des Photolyseproduktes von 3a zeigen eine gute Übereinstimmung hinsichtlich Lage und Intensität mit denen der Verbindungen $\mathbf{2 a}$ und $\mathbf{2} \mathbf{b}$.

Bei der Diskussion der Spektren (Abb. 1) kann man für die Moleküle 2d, 2a und 2b ein sehr ähnliches Schwingungsverhalten erwarten, da in diesen gleichstrukturierten Spezies von 38 Atomen nur jeweils eines ersetzt wird. Diese Erwartung entspricht dem experimentellen Befund. Da das substituierte Atom Teil eines Vierringes ist, sollten hauptsächlich Veränderungen der Ringschwingungen beobachtet werden. Darüberhinaus sind nur dann Frequenzänderungen in den Spektren zu erwarten, wenn Schwingungsbewegungen angrenzender Gruppen hinsichtlich ihrer Energie mit den Ringschwingungen vergleichbar sind. Dies ist für die $\mathrm{SiC}_{2}$-Valenz- und die $\mathrm{Si}\left(\mathrm{CH}_{3}\right)_{2}$-Rockingschwingung der Fall.

Wenn man in einer ersten Näherung die schwierigen Kopplungsverhältnisse zwischen den $\mathrm{SiN}_{2} \mathrm{M}$-Ringschwingungen, den $\mathrm{SiC}_{2}$-Valenzschwingungen und den Rockingbewegungen der Si-Methyl-Gruppen ${ }^{[10-12]}$ vernachlässigt, lassen sich in einer vereinfachten Zuordnungsbetrachtung die Frequenzänderungen der Verbindungen 2d, 2a und $\mathbf{2 b}$ im Bereich von $\tilde{v}=900 \mathrm{~cm}^{-1[13]}$ wie folgt plausibel machen: Die intensiven Banden bei $\tilde{v}=883$ (2d), 853 (2a) und $845 \mathrm{~cm}^{-1}$ (2b) müssen den in der Regel starken Absorptionen der Rockingbewegungen der $\mathrm{Si}\left(\mathrm{CH}_{3}\right)_{2}$-Gruppe zugeordnet werden. Die Frequenz dieser Normalschwingung wird offensichtlich durch Beimischung von Ringschwingungen beeinflußt, so daß die frequenzhöchste Absorption für das Silylen, die frequenzniedrigste für das Stannylen beobachtet wird.

Die Banden bei $\tilde{v}=830(\mathbf{2 d}), 814(\mathbf{2 a})$ und $811 \mathrm{~cm}^{-1}(\mathbf{2 b})$ ordnen wir aufgrund ihrer geringen Intensität $\mathrm{SiC}_{2}$-Valenzschwingungen zu. Die unterschiedlichen Frequenzen in den drei Verbindungen sind auf unterschiedliche Kopplungen mit den Ringschwingungen zurückzuführen. Die am meisten rotverschobene Absorption wird demnach folgerichtig bei der Sn-Verbindung $\mathbf{2 b}$ beobachtet.

Die Absorptionen bei $\tilde{v}=781$ (2d), 771 (2a) und $764 \mathrm{~cm}^{-1}$ (2b) sollten den überwiegenden Charakter von $\mathrm{SiN}_{2}$-Valenzschwingungen haben. Infolge der unterschiedlichen Beeinflussung durch $\mathrm{SiN}_{2}$-Schwingungen wird somit die frequenzhöchste Absorption für das Silylen, die frequenzniedrigste für das Stannylen gemessen. Die in der Reihenfolge $\mathbf{2 d} \rightarrow \mathbf{2 a} \rightarrow \mathbf{2 b}$ steigende Intensität dieser Banden wird durch die gleiche Abfolge wachsender Polarität der $\mathrm{SiN}_{2}$ Gruppe plausibel.

Die Analyse der Spektren in Abb. 1 zeigt demnach, daß bei der Photolyse der Verbindung 3a das Bis(amino)silylen 2d entstanden ist. Beim Aufwärmen und Entfernen des Argons im Vakuum ist dieses Bis(amino)silylen bis zu einer Temperatur von ca. $77 \mathrm{~K}$ stabil. Bei weiterer Erwärmung ändert sich das IR-Spektrum deutlich ${ }^{[14]}$. Im Vergleich zur analogen Photolyse von Bis(tert-butyl)silyldiazid, die zu mehreren Primärprodukten führt ${ }^{[8]}$, wovon Di-tert-butyl-silylen nur eine der Komponenten darstellt, ist die hier vorgestellte Reaktion wesentlich einfacher und einheitlicher. Auch eine Silaimin-Bildung in Analogie zur Photolyse von 
2,2-Diazidohexamethyltrisilan ${ }^{[15]}$ wird nicht beobachtet. Offenbar begünstigt die cyclische Struktur das Entstehen und die relative Stabilität des Bis(amino)silylens 2d.

\section{Experimenteller Teil}

Alle Reaktionen und Untersuchungen wurden unter AusschluB von Luft und Feuchtigkeit unter Schutzgasatmosphäre ausgeführt. - IR: Perkin-Elmer PE-597, Bruker 113 v (FT). - ${ }^{1}$ H-NMR: Bruker WP 80. - MS: Finnigan-MAT. - Elementaranalyse: Mikroanalytisches Laboratorium Beller, Göttingen.

Darstellung von 4: $0.75 \mathrm{~g}(2.40 \mathrm{mmol}) 3 \mathrm{a}$ werden in $25 \mathrm{ml} \mathrm{Benzol}$ zunächst $12 \mathrm{~h}$ mit einer Hg-Niederdrucklampe [TNN 15/32 (15 Watt), Firma Heraeus, Hanau], dann $12 \mathrm{~h}$ mit einer Hg-Hochdrucklampe ( $80 \mathrm{~W}$, gleiche Firma) bestrahlt. Der äußere Mantel der Photolyseapparatur wird dabei auf $5^{\circ} \mathrm{C}$ gekühlt. Nach Abkondensieren des Lösemittels erhält man einen bernsteinfarbenen Feststoff, der röntgenamorph ist (Pulvermethoden). Die entstandene $\mathrm{N}_{2-}$ Menge wird durch Differenzwägung (das Gasvolumen konnte nur geschätzt werden) ermittelt (berechnete Werte für $3 \mathrm{~N}_{2}$ in Klammern): $190 \pm 10$ (201.7) mg (siehe auch Elementaranalyse von 4) - IR [Benzol (kompensiert)]: $\tilde{v}=2980 \mathrm{~cm}^{-1}$ (s), 2940 (s), 1365 (m), 1250 (Sh, m), 1225 (s), 1090 (s), 1040 (Sh, w), 930 (Sh, w), 885 (vs), $840(\mathrm{~m}), 780(\mathrm{w}) .-{ }^{1}$ H-NMR [Benzol (Integrationen in Klammern)]: mehrere Linien bei $\delta=0.5$ (ca. 1.0) und 1.4 (ca. 3.0). MS $\left(40 \mathrm{eV} ; 55^{\circ} \mathrm{C}\right.$, Massen > 200): $m / z(\%)=243(16)$ [ber. $\mathrm{C}_{11} \mathrm{H}_{27} \mathrm{~N}_{2} \mathrm{Si}_{2}, \mathrm{M}^{+}-15$ ], 471 (5) [ber. $\mathrm{C}_{21} \mathrm{H}_{51} \mathrm{~N}_{4} \mathrm{Si}_{4}$ ], 499 (5) [ber. $\mathrm{C}_{21} \mathrm{H}_{51} \mathrm{~N}_{4} \mathrm{Si}_{5}$ ], 642 (6).

$$
\mathrm{C}_{10} \mathrm{H}_{24} \mathrm{~N}_{2} \mathrm{Si}_{2} \cdot 0.6 \mathrm{C}_{6} \mathrm{H}_{6}(275.36) \quad \text { Ber. C 59.32 H } 10.10 \mathrm{~N} 10.17
$$

$$
\text { Gef. C } 61.73 \text { H } 10.48 \text { N } 10.58
$$

Photolyse von 3a: Den Kryostaten und die Aufdampfbedingungen haben wir bereits früher beschrieben ${ }^{[16]}$. Die IR-Spektren wurden mit einem FT-IR-Gerät in Absorption in einer Reflexionsanordnung gemessen. Die Verbindung 3a wird zusammen mit Argon auf eine $\mathrm{Cu}-\mathrm{Kühlfläche} \mathrm{abgeschieden} \mathrm{(Verhältnis} \mathrm{1:200)} \mathrm{und}$ mit dem Gesamt-Spektrum einer Hg-Hochdrucklampe (Osram HBO 200) bestrahlt. Nach 10min. Belichtung ist IR-spektroskopisch kein Edukt mehr nachweisbar (z. B. vollständiges Verschwinden der Azid-Banden von 3a). - IR-Spektren von 2d (Argon-Matrix; s. Abb. 1), 2a (Film) und 2 b (Film):

2d: $\tilde{v}=1463 \mathrm{~cm}^{-1}(\mathrm{~m}), 1402(\mathrm{w}), 1389(\mathrm{w}), 1363,1361(\mathrm{w}), 1252$ (s), 1241 (m), 1226 (vs), $1112(\mathrm{~m}), 1081$ (vs), 1038 (m), 898 (m), 883 (s), $830(\mathrm{~m}), 781(\mathrm{~m})$. 2a: $\tilde{v}=1457 \mathrm{~cm}^{-1}(\mathrm{~m}), 1396(\mathrm{w}), 1382(\mathrm{w}), 1356(\mathrm{~s}), 1245(\mathrm{~s}), 1216$ (vs), 1105 (m), 1095 (m), 1055 (vs), 1032 (m), 889 (m), 853 (vs), 814 (m), $787(\mathrm{~s}), 771(\mathrm{~s}), 709(\mathrm{w}), 675(\mathrm{w})$

2b: $\tilde{v}=1457 \mathrm{~cm}^{-1}(\mathrm{~m}), 1396(\mathrm{w}), 1378(\mathrm{~m}), 1354(\mathrm{w}), 1242$ (s) 1221 (Sh), 1209 (vs), 1088 (m), 1047 (vs), 1027 (m), 845 (vs), 811 (m), $764(\mathrm{vs}), 698(\mathrm{w}), 670(\mathrm{w})$.

${ }^{[1]}$ W. Fink, Helv. Chim. Acta 1964, 47, 498

${ }^{[2]}{ }^{[2 a]}$ M. Veith, Angew. Chem. 1975, 87, 287; Angew. Chem. Int. Ed. 1975, 14, 263. - ${ }^{[26]}$ M. Veith, M. Grosser, V. Huch, Z. Anorg Allg. Chem. 1984, 513, 89 .

${ }^{[3]}$ P. Jutzi, U. Holtmann, D. Kanne, C. Krüger, R. Blome, G. Gluter, J. Hyla-Krispin, Chem. Ber. 1989, 122, 1629.

${ }^{[4]}$ H. Karsch, U. Keller, S. Gamper, G. Müller, Angew. Chem. 1990, 102, 297; Angew. Chem. Int. Ed. Engl. 1990, 29, 295.

${ }^{[5]}$ Im strengen Sinne können diese Moleküle allerdings nicht als Silylene bezeichnet werden, da nach einer allgemeinen Definition bei Carben-Analogen nur zwei Bindungen vom Elektronenmangelzentrum ausgehen sollten (Koordinationszahl 2 am Silicium-Atom): ${ }^{[5 a]}$ M. E. Kolpin, Y. D. Kerelikov, V. G. Zulova, D. N. Kursanov, Tetrahedron 1962, 18, 107. - ${ }^{[5 b]}$ O. M. Nefedov, M. N. Manakov, Angew. Chem. 1966, 78, 1039; Angew. Chem. Int. Ed. Engl. 1966, 5, 1021.

${ }^{[6]}$ M. Veith, R. Lisowsky, Z. Anorg. Allg. Chem. 1988, 560, 59.

${ }^{77}$ M. Veith, E. Werle, Z. Anorg. Allg. Chem., im Druck.

${ }^{[8]}$ K. M. Welsh, J. Michl, R. West, J. Am. Chem. Soc. 1988, 110, 6689.

${ }^{[9]}$ H. Bock, R. Dammel, Angew. Chem. 1987, 99, 518. Angew. Chem. Int. Ed. Engl. 1987, 26, 504.

${ }^{[10]}$ H. Schnöckel, L. Zhengyan, N. Auner, P. Bleckmann, M. Hinrichsen, J. Mol. Struct. 1985, 127, 1.

${ }^{[11]}$ G. Fritz, W. Kemmerling, G. Sonntag, H. J. Becher, E. A. V Ebsworth, J. Grobe, Z. Anorg. Allg. Chem. 1963, 321, 10.

${ }^{[12]}$ T. Mehner, H. J. Göcke, S. Schunck, H. Schnöckel, Z. Anorg. Allg. Chem. 1990, 580, 121.

${ }^{[13]}$ Bei $\tilde{v}>900 \mathrm{~cm}^{-1}$ liegen nur die Absorptionen der $\mathrm{C}-\mathrm{C}$ Schwingungen und der $\mathrm{CH}_{3}-\mathrm{Gruppen}$. Ihre Frequenzen werden erwartungsgemä $ß$ nur geringfügig durch die Substitution im $\mathrm{SiN}_{2} \mathrm{M}$-Ring beeinflußt.

${ }^{[14]}$ Die entstehenden Produkte, z. B. bei der Reaktion der SilylenMoleküle untereinander enstehende Spezies, konnten bisher noch nicht identifiziert werden.

${ }^{[15]}$ S. S. Zigler, K. M. Welsh, J. Michl, R. West, J. Am. Chem. Soc. 1987, 109, 4392.

${ }^{[16]}$ R. Ahlrichs, R. Becherer, M. Binnewies, H. Borrmann, M. Lakenbrink, S. Schunck, H. Schnöckel, J. Am. Chem. Soc. 1986, 108,7905

[23/92]

\section{CAS-Registry-Nummern}

2a: $84806-15-5$ / 2b: 54724-62-8 / 2d: 140149-34-4 / 3a: $120592-$ 13-4/4: 140149-35-5 